\title{
Time to rethink asthma management
}

\section{Richard E Ruffin}

From around the world we have had evidence that the "guideline-driven" attainment of good asthma control is not being achieved. ${ }^{1-3}$ Surveys over the past decade have provided the evidence and concluded that "physicians and patients should raise their expectations of the level of asthma control that can be achieved", 2 and "the critical need for improved asthma care, including a more global evaluation of asthma control, implementation of asthma treatment plans and addressing co-morbid conditions". "In this issue of Thorax the article by Kandane-Rathnayake et $a l^{4}$ describes the loss of lung function in middle-aged patients not using inhaled corticosteroids for their asthma (see page 1025). This study adds to the survey evidence ${ }^{1-3}$ by providing lung function data in a population that has been part of an asthma cohort study for several decades. It is a timely wake-up call that, in spite of having doctor-agreed guidelines for asthma management available for two decades, ${ }^{5}$ predominantly in response to asthma mortality crises in some countries, we still have major ongoing morbidity with asthma and loss of function occurring in a significant proportion of patients with asthma. We have medications which are fit for the task of controlling asthma in most patients and, although medication improvements will occur over time, this is likely to be of small impact in the total scheme of asthma management in the community. We need to question why there is the gap between the guideline ambitions of good control and the patient outcome. Is it related to:

- patients and their attitudes including fear or dislike of medications and/or not believing the diagnosis;

- access to the healthcare system;

- access to medications;

- limited time of health professionals to put into asthma care;

- fragmentation of care of chronic asthma;

Correspondence to: Professor R E Ruffin, Discipline of Medicine, University of Adelaide, Queen Elizabeth Hospital, Woodville, South Australia, Australia; richard. ruffin@adelaide.edu.au
- economic factors with work schedules as well as medication costs; or

\section{- psychosocial factors?}

It is likely that in different countries there will be different reasons and between individual patients there will also be different reasons accounting for overall poor asthma control. In other words, it seems unlikely that one set of reasons is going to explain the variation between individuals or between countries to enable a single new plan to be developed which will overcome all possible factors inhibiting an ideal asthma outcome.

So it is time to re-evaluate and flexibly add to the solid framework that asthma guidelines now provide for asthma management. Previous studies have provided pointers that need to be addressed in such a re-evaluation. Some of these are:

- Some people do not accept they have asthma. ${ }^{6}$

- Non-compliance ${ }^{7}$ or non-adherence ${ }^{4}$ to prescribed medications is a frequent occurrence in asthma.

- For most patients, asthma treatment goals are not the most important factor $^{8}$ because the experience of asthma is not seen as a problem.

- Avoidance coping style and socioeconomic factors are associated with a reduced quality of life in patients with asthma. ${ }^{9}$

- Denial is associated with suboptimal compliance. $^{10}$

- Psychosocial factors indicate barriers to self-management. ${ }^{11}$

- Inadequate health literacy increases asthma morbidity. ${ }^{12}$

Other authors have indicated important content for future asthma management strategies: communication improvement between the health professional and the patient with asthma; ${ }^{13}$ identifying and addressing patient-related reasons for poor asthma control and taking into account the patient perspective during asthma care. ${ }^{14}$

The Global Initiative for Asthma (GINA) ${ }^{15}$ contains discussion about broad principles of adherence and developing patient/doctor relationships but does not operationalise these principles for the clinical situation.

It seems that progress in asthma control will require a flexible multidisciplinary approach with opportunity and time for communication ${ }^{16}$ between disciplines and with patients to achieve optimum outcomes for individual patients and the community. This may require professional barriers to be lowered in some countries and will require sustainable funding, possibly linked to patient outcomes.

Models exist for pharmacy-led ${ }^{17}$ and nurse-led $^{18}$ asthma care and nurse-led home-based interventions. ${ }^{19} \quad$ The Australian Government has shown that it recognises the need for improved asthma outcomes by funding asthma programmes in general practice via practice incentive programmes (Asthma 3+ visit plan and now Asthma Cycle of Care).$^{20}$ The next step is to recapture the enthusiasm of the early 1990s for asthma management and to nationally discuss with stakeholders the methods to provide optimal asthma outcomes with flexible processes for the patient with asthma. We have ample evidence that we need to add to current management strategies. Can we devise innovative flexible programmes in each country which will be sustainable? Yes, provided we go forward with communication, cooperation, goodwill and in the knowledge that major advances were made by the initial guidelines (national and global).

The article by Kandane-Rathnayake et $a l^{4}$ also reminds us to adopt a holistic patient management approach. In their group with severe asthma, 40\% were smokers-a recipe for poor asthma outcomes!

\section{Competing interests: None.}

Provenance and peer review: Commissioned; not externally peer reviewed.

Thorax 2009;64:1013-1014.

doi:10.1136/thx.2009.124040

\section{REFERENCES}

1. Rabe KF, Vermeire PA, Soriano JB, et al. Clinical management of asthma in 1999: the Asthma Insights and Reality in Europe (AIRE) study. Eur Respir $J$ 2000;16:802-7.

2. Rabe KF, Adachi M, Lai CKW, et al. Worldwide severity and control of asthma in children and adults: the global Asthma Insights and Reality surveys. J Allergy Clin Immunol 2004;114:40-7.

3. Peters SP, Jones CA, Haselkorn T, et al. Real-world Evaluation of Asthma Control and Treatment (REACT): findings from a national web-based survey. J Allergy Clin Immunol 2007;119:1454-61.

4. Kandane-Rathnayake RK, Matheson MC, Simpson $\mathrm{JA}$, et al. Adherence to asthma management 
guidelines by middle-aged adults with current asthma. Thorax 2009;64:1025-31.

5. Woolcock A, Rubinfeld AR, Seale JP, et al. Thoracic Society of Australia and New Zealand Asthma Management Plan 1989. Med J Aust 1989;151: 650-3.

6. Adams S, Pill R, Jones A. Medication, chronic illness and identity: the perspective of people with asthma. Soc Sci Med 1997;45:189-201.

7. Dekker FW, Dieleman FE, Kaptein AA, et al. Compliance with pulmonary medication in general practice. Eur Respir J 1993;6:886-90.

8. Steven K, Morrison J, Drummond N. Lay versus professional motivation for asthma treatment: a cross-sectional, qualitative study in a single Glasgow general practice. Fam Pract 2002;19:172-7.

9. Adams RJ, Wilson D, Smith BJ, et al. Impact of coping and socioeconomic factors on quality of life in adults with asthma. Respirology 2004;9: 87-95.
10. McGann EF, Sexton D, Chyun DA. Denial and compliance in adults with asthma. Clin Nurs Res 2008;17:151-70.

11. Rhee H, Belyea MJ, Ciurzynski S, et al. Barriers to asthma self-management in adolescents: relationships to psychosocial factors. Pediatr Pulmonol 2009;44:183-91.

12. Adams RJ, Appleton SL, Hill CL, et al. Inadequate health literacy is associated with increased asthma morbidity in a population sample. J Allergy Clin Immunol 2009. doi:10.1016/j.jaci.2009.05.035

13. Partridge MR. Living with a variable disease. Pulm Pharmacol Ther 2002:15:491-6.

14. Horne R, Price D, Cleland J, et al. Can asthma control be improved by understanding the patient's perspective? BMC Pulm Med 2007;7:8.

15. Global Initiative for Asthma (GINA). Global strategy for asthma management and prevention. Updated 2008. www.ginasthma.org (accessed 17 Aug 2009).
16. Charlton I, Charlton GF. Caring for patients with asthma. Teaching self management takes time. BMJ 1994;308:1370-1.

17. Saini B, Krass I, Armour C. Development, implementation, and evaluation of a community pharmacy-based asthma care model. Ann Pharmacother 2004;38:1954-60.

18. Charlton I, Charlton G, Broomfield J, et al. Audit of the effect of a nurse run asthma clinic on workload and patient morbidity in a general practice. $\mathrm{Br} \mathrm{J}$ Gen Pract 1991;41:227-31.

19. Smith JR, Mildenhall S, Noble MJ, et al. The Coping with Asthma Study: a randomised controlled trial of a home based, nurse led psychoeducational intervention for adults at risk of adverse asthma outcomes. Thorax 2005;60:1003-11.

20. Australian Government Medicare Australia Practice Incentives Program (PIP) - Asthma Incentive. http://www.medicareaustralia.gov.au/provider/ incentives/pip/payment-formula/asthma-incentive.jsp (accessed 19 Aug 2009)

\section{Asthma: what's the bleeding point?}

\section{Simon C Pitchford, ${ }^{1}$ James D Moffatt ${ }^{2}$}

Inflammation and coagulation are complex biological pathways that interact with each other in a variety of chronic diseases. Although they are often considered to be distinct processes, it is almost impossible to dissect the one from the other, except at their functional extremes. ${ }^{1}$ Activation of the coagulation cascade appears to be common to diverse inflammatory lung diseases such as acute lung injury, idiopathic lung fibrosis, acute respiratory distress syndrome and sarcoidosis. ${ }^{2}$ It is no great surprise, then, to find evidence of activation of the coagulation cascade in a chronic inflammatory disease such as asthma. Indeed, several studies over the last 10 years or so have consistently found markers for coagulation in the airways of subjects with asthma. ${ }^{3-7}$ However, taking an informative snapshot of all the relevant team members in coagulation and inflammation in such a setting is a bit like taking a photograph of a 10-car pile-up on a motorway. Such pictures could generate widespread speculation as to the true course of events that led to the disaster. A comprehensive exploration of the presence of various

\footnotetext{
${ }^{1}$ Leukocyte Biology Section, National Heart and Lung Institute, Imperial College London, London, UK; ${ }^{2}$ Division of Basic Medical Sciences, St George's, University of London, London, UK

Correspondence to: Dr Simon Pitchford, Leukocyte Biology Section, National Heart and Lung Institute, Imperial College London, 3rd Floor, Sir Alexander Fleming Building, Exhibition Rd, London SW7 2AZ, UK; s.pitchford@imperial.ac.uk
}

factors governing the balance between coagulation and fibrinolysis, and their relationship to asthma severity has been lacking. In this issue of Thorax, Brims et al (see page 1037) provide a new, more complete montage of the potential family members of the coagulation cascade present in the asthmatic airway. ${ }^{8}$ What can we learn from it, and what future work would help us see how inflammatory pileups may be modulated or prevented?

Although glucocorticosteroids are the mainstay of asthma treatment, many of the structural changes that occur in the airways appear to be steroid resistant. ${ }^{9}{ }^{10}$ It is likely, therefore, that some remodelling processes occur independently of inflammation. In subjects with severe asthma, Brims et al reveal that procoagulant factors which might contribute to airway remodelling (see below) persist at elevated levels despite glucocorticosteroid therapy, ${ }^{8}$ and are independent of inflammatory cell influx. ${ }^{8}$ These extrinsic procoagulant factors are probably produced by a local cellular source (eg, epithelium, macrophages or fibroblasts) rather than from circulating cells. ${ }^{11}{ }^{12}$ Additionally, there is increasing evidence of platelet activation in patients with asthma. ${ }^{13}$ Platelets have also been observed to penetrate through lung interstitial tissue directly in response to allergen and locate to the airway wall. ${ }^{14}$ Because they lack a nucleus, platelets are one of the few cells in the body that are unaffected by glucocorticosteroids.
Further, platelet depletion resulted in the virtual abolition of remodelling events in a murine model of chronic allergic inflammation, whereas some remodelling events (including subepithelial fibrosis and collagen deposition) persisted after continuous administration of a glucocorticosteroid (dexamethasone). ${ }^{15}$ Extravascular platelets may therefore also provide a potent stimulatory platform for coagulatory events leading to airway remodelling in the lungs of subjects with asthma that is steroid resistant.

How would mediators of the coagulation/fibrinolytic processes affect the lung? Brims et al suggest that in subjects with moderate asthma there may be a fibrinolytic environment that encourages destruction of the extracellular matrix (ECM) via enhanced plasmin production (eg, through increased tissue plasminogen activator (tPA) and urokinase plasminogen activator ( $\mathrm{UPA}$ ) release, inhibition of plasminogen activator inhibitor-1 (PAI-1) or activation of protein $C$ ) ensuring the removal of glycoproteins and activation of matrix metalloproteinases (MMPs). ${ }^{8}$ It has been hypothesised that inappropriate activation of this plasmin system would accelerate the production of inflammatory mediators and growth factors that influence processes such as collagen deposition and airway smooth muscle (ASM) proliferation. ${ }^{16}$ However, it is difficult to envisage this fibrinolytic scenario occurring in the lungs purely on the evidence gained with this clinical study. Alternatively, the profibrinogenic/antifibrinolytic environment reported here in subjects with severe asthma and also in those with moderate asthma directly after allergen exposure might affect lung function via fibrin deposition or via a soup of coagulation factors involved in remodelling. ${ }^{3} 51718$ 